\title{
Pierre Gisel, Traiter du religieux à l'université. Une dispute socialement révélatrice
}

Lausanne, Antipodes, coll. « Contre-pied », 2011, 174 p.

Pierre Lassave

\section{OpenEdition}

Journals

Édition électronique

URL : https://journals.openedition.org/assr/23980

DOI : $10.4000 /$ assr.23980

ISSN : $1777-5825$

Éditeur

Éditions de l'EHESS

Édition imprimée

Date de publication : 30 décembre 2012

Pagination : 195

ISSN : 0335-5985

Référence électronique

Pierre Lassave, "Pierre Gisel, Traiter du religieux à l'université. Une dispute socialement révélatrice », Archives de sciences sociales des religions [En ligne], 160 | octobre-décembre 2012, mis en ligne le 13 mars 2013, consulté le 21 septembre 2021. URL : http://journals.openedition.org/assr/23980 ; DOI : https://doi.org/10.4000/assr.23980

Ce document a été généré automatiquement le 21 septembre 2021.

(c) Archives de sciences sociales des religions 


\section{Pierre Gisel, Traiter du religieux à l'université. Une dispute socialement révélatrice}

Lausanne, Antipodes, coll. « Contre-pied », 2011, 174 p.

\section{Pierre Lassave}

\section{RÉFÉRENCE}

Pierre Gisel, Traiter du religieux à l'université. Une dispute socialement révélatrice, Lausanne, Antipodes, coll. « Contre-pied », 2011, 174 p. 
1 Il arrive encore dans la vieille Europe sécularisée que la grande presse s'intéresse à ce qui reste de l'ancien conflit des facultés entre théologie et philosophie. Ce fut le cas en Suisse romande dans les années 2007-2008 quand un projet de réorganisation de

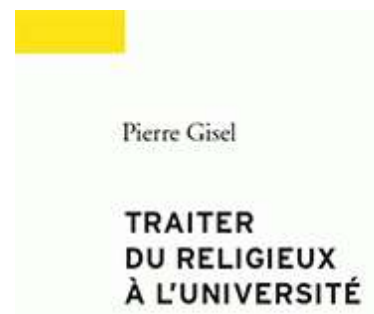
l'enseignement conjoint de la théologie dans les facultés de Lausanne, de Genève et de Neuchâtel suscita une dispute universitaire mémorable. Pierre Gisel, professeur de théologie protestante connu pour ses thèses libérales et principal acteur des événements, nous livre ici une chronique de cet épisode mouvementé. Un témoignage de première main à la fois documenté, réflexif et engagé sur un conflit académique local dont les lignes de force et de fuite dépassent le seul cas suisse.

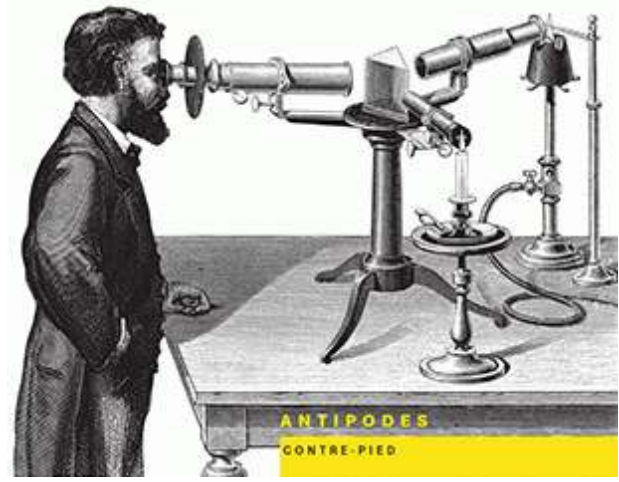

2 Rappelons quelques chiffres pour planter

le décor : à la fin des années 1970, la faculté de théologie de Lausanne accueillait chaque année près d'une vingtaine d'étudiants dans une petite unité logée au sein d'une université multidisciplinaire de quatre milles inscrits; vingt ans plus tard, elle en devient encore plus minuscule en n'accueillant qu'une dizaine d'étudiants au mieux dans une université dont les effectifs ont triplé. Les facultés protestantes de théologie forment depuis l'origine ses étudiants à la profession de pasteur en délivrant une solide formation biblique dans les langues sources et en inculquant la maîtrise de la doctrine et de la mission chrétiennes. Mais la crise des vocations pastorales étonnamment concomitante de la résurgence des questions religieuses dans le monde infléchit depuis quelques années la demande de formation universitaire dans le sens des sciences humaines. Celles-ci prennent le fait religieux comme un phénomène observable et objectif n'engageant plus nécessairement le sujet de la connaissance de façon existentielle. Dans ce contexte, l'impératif gestionnaire d'adaptation des moyens aux missions universitaires nouvelles est à Lausanne comme ailleurs à l'origine de réformes récurrentes. Il en fut ainsi du transfert des sciences exactes (physique, chimie, mathématiques) à l'École polytechnique fédérale de Lausanne et du renforcement en retour de l'université en matière de sciences criminelles, de biotechnologies médicales ou de sciences de l'environnement. L'économie de moyens dans le "Triangle Azur » (universités de Genève, Lausanne et Neuchâtel) rapproche également les filières d'enseignement de la théologie selon des conventions entre facultés qui ne tardent pas à faire ressortir les spécialités respectives. Lausanne apparaît ainsi pilote en matière d'ouverture ou d'association de la théologie aux sciences des religions fondées sur l'histoire, la sociologie et l'anthropologie. Regroupement scientifique autour d'un objet commun qui ne cesse de se développer à la marge des universités littéraires d'Europe depuis le XIX ${ }^{e}$ siècle. La section de « sciences religieuses » de l'École pratique des hautes 
études en France en fournit ainsi un exemple d'érudition et de souci comparatiste entre les multiples traditions spirituelles du monde.

3 La chronique lausannoise commence par le rappel des étapes de cette association locale entre théologie et sciences des religions, notamment l'appellation officielle de « Faculté de théologie et de sciences des religions» en 2006 après plusieurs années de négociations au sein du corps enseignant. Une faculté qui fait cohabiter en clair trois sections: les sciences bibliques, les sciences des religions et un observatoire sociologique de la modernité religieuse. Les choses se tendent un an plus tard lorsque le rectorat épris de rigueur budgétaire échafaude des scénarios divers où la théologie se trouve regroupée à Genève et les sciences des religions à Lausanne ou bien ces dernières placées sous la dépendance théologique du Triangle Azur. Notre auteur, alors professeur de théologie à Lausanne, saisit là l'occasion pour engager un débat sur le fond de ce que peut être un enseignement de la théologie chrétienne au temps des sciences des religions renaissantes et du dialogue progressif entre traditions croyantes du monde globalisé. Il avance l'idée de "scène religieuse» comme objet de connaissance se référant à une pluralité d'énoncés et d'acteurs. Sur cette scène, la théologie ne figure plus comme discipline rectrice du rapport avec l'ultime, mais comme tradition de pensée dont les contours et les développements suivent le cours particulier de la civilisation occidentale. Cette dernière, de sources gréco-judéoromano-chrétiennes, a précisément inventé le mot « religion » à la différence d'autres cadres de pensée qui ne l'ont pas toujours distingué. Poser la scène religieuse comme objet justiciable d'une approche généalogique attentive autant aux ruptures qu'aux continuités conduit à remettre en question les catégories héritées, telles que les oppositions dualistes entre science et théologie, sacré et profane, tradition et modernité. La distinction monothéiste entre une transcendance unitaire et une immanence plurielle se trouve ainsi déconstruite en chaos conjuré pour le judaïsme, en mystère habité pour le christianisme ou en illusion révoquée pour l'athéisme, «vie nue "(Giorgio Agamben) livrée aux «biopouvoirs»(Michel Foucault). Pour Gisel, «l'humain » en sa propension à la symbolisation absolue de l'être au monde s'avère plus que le divin l'horizon d'attente actuel de l'intelligence de la foi. Telle hypothèse ne va pas, on s'en doute, sans conséquence programmatique au plan universitaire: la théologie chrétienne ne peut plus dès lors rester le cadre impensé et invariable d'une relation datée au divin. L'auteur tend ainsi à « désanctuariser » le savoir théologique en le réinsérant dans l'histoire culturelle des sociétés. La déconstruction des textes canoniques qui retrace leur pluralité constitutive et leurs compromis négociés, non seulement désacralise la collection biblique, mais déstabilise aussi le privilège accordé à tels livres au détriment de ceux que les conciles ont décrété apocryphes.

4 La réaction de ses collègues théologiens ne s'est pas faite attendre. Denis Müller, par exemple, ne se satisfait pas de cette relégation du potentiel critique de la théologie protestante au musée de la pensée occidentale. Pour lui, «La théologie universitaire a pour tâche de problématiser l'usage du mot "Dieu" et de rendre palpable la querelle incessante des dieux et des idoles qui anime le temps présent. Solidaire de toute recherche libre et de tout savoir critique, elle fait et dit autre chose que la philosophie, les sciences sociales et historiques ou les sciences des religions, ne cessant d'interroger radicalement la société sur les questions de vie et de mort, de mal radical, de justice, d'amour et d'espérance » (p. 85, citation d'un article paru dans le quotidien genevois Le Temps en mars 2008). Pour les tenants des sciences des religions, une telle prise de position publique fait craindre pour l'indépendance de l'histoire ou de la philosophie 
en régime de faculté théologique. La réaction en chaîne se poursuit. Ces enseignantschercheurs menacent ainsi de quitter la faculté lausannoise pour rejoindre les facultés littéraires ou de sciences politiques. L'une d'entre eux est d'ailleurs passée à l'acte.

Lancée en 2007, la controverse universitaire a ainsi trouvé écho dans la grande presse en 2008. L'auteur nous livre son épais dossier. Son commentaire vise le dépassement nécessaire des antagonismes exposés au grand jour. Lui-même en tant qu'acteur central propose une sorte de compromis en avançant l'idée d'une double polarisation des savoirs dans le Triangle Azur : à Genève l'étude du christianisme, à Lausanne celle de la scène religieuse universelle, à Neuchâtel la formation proprement pastorale et missionnaire. Et pour joindre le geste à l'intention, le théologien demande son rattachement direct à la section de sciences des religions de la faculté lausannoise. Vœu peu après accordé par ses pairs avec un changement d'intitulé de la chaire qui est significatif: on passe ainsi de l'enseignement de la «Dogmatique et théologie fondamentale » à celui d'« Histoire des théologies, des institutions et des imaginaires chrétiens ». Comme on le devine, la double polarisation préconisée entre Lausanne et Genève ne va pas de soi dans la mesure où les compétences des deux côtés demeurent largement structurées par l'histoire de la formation théologique avec ses trois niveaux plus ou moins emboîtés entre eux : les sciences bibliques, la théologie systématique et la théologie pastorale. De plus, dans ce pays de laïcité concordataire les intérêts ecclésiaux interfèrent avec les intérêts universitaires (l'université de Genève est liée à une fondation confessionnelle; la hiérarchie protestante a droit de regard sur les programmes théologiques et la nomination de leurs enseignants). Au terme de débats publics et de négociations universitaires mettant aux prises les logiques gestionnaires, ecclésiales, épistémologiques, disciplinaires et personnelles s'esquisse une forme de compromis à l'automne 2008 : le pôle théologique lausannois voit son association avec les sciences des religions renforcée et celui de Genève conforté dans son orientation fondamentale et systématique. Si la séparation entre théologie et sciences des religions n'est plus à l'ordre du jour, les frontières programmatiques ont cependant bougé. Ainsi se crée à Lausanne, outre la chaire de notre théologien, un Institut « Religions, cultures, modernité », pôle de recherche tourné vers la diversité des acteurs de la scène religieuse dans le monde contemporain qu'il s'agisse d'autres traditions que le christianisme ou de la transformation actuelle de chacune d'entre elles dans un contexte d'interférences culturelles accrues. Les recrutements à la marge d'enseignants-chercheurs et de doctorants suivent cette extension du domaine savant qui vient ainsi compenser celui propre à une formation pastorale sur le déclin et dont une partie d'ailleurs est directement assurée par les dénominations respectives (tendances évangéliques) hors de l'enceinte académique.

6 Tirant le bilan de ces modifications, l'auteur conclut à ce qu'il appelle un "projet avorté », celui de la refondation des disciplines nouvelles et anciennes autour d'un objet commun, la fameuse scène religieuse. Son idéalisme transparait quand il déplore que ses propositions n'aient pu trouver leur justification dans un débat partagé et argumenté sur le fond. On peut cependant supposer qu'il n'est pas dupe des contingences et obstacles mentaux, existentiels et institutionnels que ses thèses ont rencontrés malgré le bout de chemin engagé à Lausanne. À la balkanisation mondiale du christianisme (essor évangélique au Sud, repli et médiatisation catholique, spiritualisme flottant au Nord) correspondrait en écho local la cote mal taillée de la réforme suisse : les sciences bibliques campant résolument dans les intervalles entre philologie, histoire et herméneutique; les sciences des religions s'affranchissant de 
leur matrice chrétienne occidentale; la théologie protestante oscillant entre critique radicale de la modernité et idéologie missionnaire.

7 Ce témoignage de première main sur un moment et un lieu universitaire particuliers s'avère doublement précieux: d'abord par la mise en ordre ou en intrigue d'un ensemble de discours éclatés et plus ou moins publics, ensuite par la portée que ce conflit contemporain des facultés a plus généralement pour qui s'intéresse au traitement universitaire des phénomènes que l'on rassemble sous le terme contesté de religions. Il a également le mérite de mettre au grand jour ce qui relève souvent de l'implicite sinon du secret des cercles d'initiés. La qualité littéraire du récit dût-elle d'ailleurs en souffrir: l'auteur abuse un peu du verbe "gérer", des expressions familières du type "à l'interne/à l'externe » ou des chevilles lourdes telles que « tant quant à...»). Mais la matière brute et déjà semi-traitée est bien au rendez-vous. L'engagement personnel de l'auteur dans sa chronique enrichit de surcroît sa valeur et paradoxalement d'ailleurs sa portée générale. On souhaiterait que d'autres témoignages de conflits significatifs puissent de la sorte éclairer le destin des disciplines savantes, théologie comprise, qui tournent autour de faits éminemment problématiques. 